\title{
LINE SHIFTS AND ASYMMETRIES IN THE \\ IR SOLAR SPECTRUM
}

\author{
R. BLOMME and A. J. SAUVAL \\ Koninklijke Sterrenwacht van België/Observatoire Royal de Belgique, \\ B-1180 Brussels, Belgium \\ and \\ N. GREVESSE \\ Institut d'Astrophysique, Université de Liège \\ 5, av. de Cointe, B-4000 Cointe-Liège, Belgium
}

\begin{abstract}
Line shifts and asymmetries of spectral lines have been found in the ATMOS spectra. These are due to the granulation at the solar surface. A two-component model for the solar photosphere is used to calculate theoretical profiles which are compared to the observations. The ATMOS lines provide additional constraints on models for the solar photosphere.
\end{abstract}

Key words: infrared: stars - Sun: granulation - Sun: photosphere

\section{Introduction}

Dravins et al. $(1981,1986)$ made a detailed study of line asymmetries observed in the Fe lines. The best way of presenting these asymmetries is through the use of the line bisector, i.e., the line connecting the points midway between the blue and red side of the spectral line at equal intensity. These bisectors show a classical "C" shape instead of the vertical line that one would expect if the line were symmetric (see Fig. 1). When the deviations of the bisector are interpreted in terms of velocity, values of up to a few hundred $\mathrm{m} / \mathrm{s}$ are observed.

Furthermore, when the laboratory wavelength of the line is known, one can compare it with the observed wavelength that has been corrected for the motion of the observer with respect to the sun, solar rotation and the gravitational redshift. The results show that the center of the line is shifted to the blue (see, e.g., Dravins et al., 1981; Nadeau, 1988).

Both phenomena can be explained as being due to the granulation present in the solar photosphere. Because the rising granules are hotter than the descending intergranular material, the Doppler shifts do not cancel exactly and an asymmetric line is formed; this is described in detail by de Jager (1959).

These asymmetries are not limited to lines in the visible region, but are also found in the infrared (see, e.g., Nadeau, 1988). In this paper we try to fit the observed bisectors using a two component model for the granulation.

\section{Observations}

Preliminary experiments with our two-component model showed that when the calculated stronger lines showed a bisector similar to the observed ones then, for the same model, so did the weaker lines. We therefore decided to concentrate on the stronger lines. In the visible part of the spectrum, we selected $3 \mathrm{Fe} \mathrm{I}$ lines and 3 Fe II lines (see Table I). 

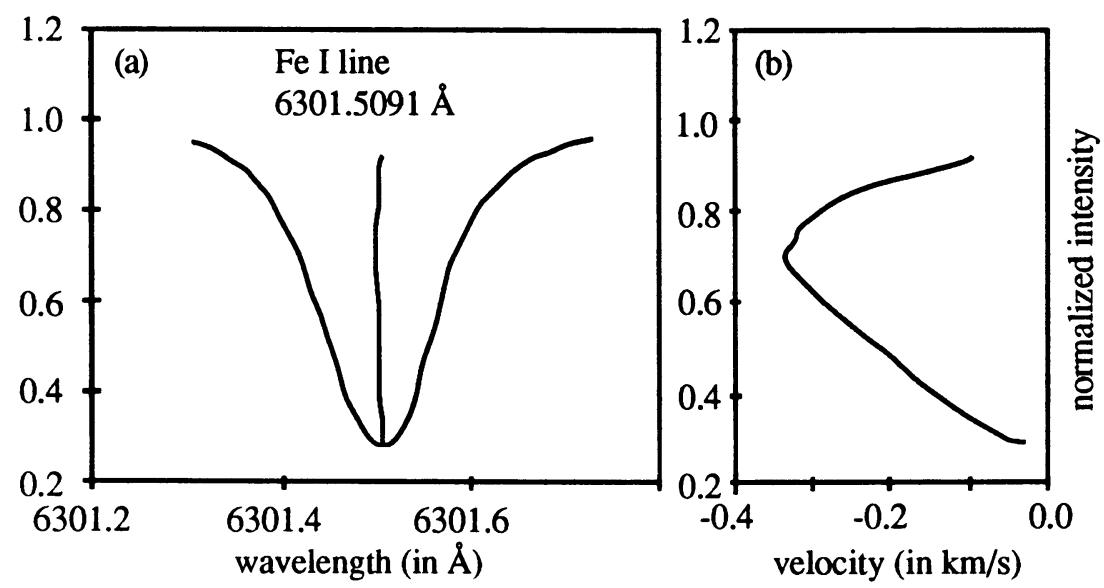

Fig. 1. (a) The observed line profile of an Fe I line (from Delbouille et al., 1973). (b) An enlargement of the bisector where the wavelength shifts have been interpreted as velocities.

TABLE I

The spectral lines used in this analysis. Solar wavelengths from Pierce and Breckinridge (1973) are given for $\mathrm{Fe}$, and calculated wavenumbers from Farrenq et al. (1991) for $\mathrm{CO}$.

\begin{tabular}{cc|cc}
\hline \multicolumn{1}{c|}{$\begin{array}{c}\text { Designation } \\
\text { Fe I }\end{array}$} & $\begin{array}{c}\text { Wavelength } \\
(\AA)\end{array}$ & Designation & $\begin{array}{c}\text { Wavenumber } \\
\left(\mathrm{cm}^{-1}\right)\end{array}$ \\
\hline & & \multicolumn{2}{|c}{$\mathrm{CO}$} \\
& & \multicolumn{2}{|c}{} \\
$\mathrm{a}^{5} \mathrm{P}_{1} \rightarrow \mathrm{y}^{5} \mathrm{D}_{2}^{0}$ & 6297.8013 & $4-3 \mathrm{R}(18)$ & 2128.2738 \\
$\mathrm{z}^{5} \mathrm{P}_{2}^{0} \rightarrow \mathrm{e}^{5} \mathrm{D}_{2}$ & 6301.5091 & $7-6 \mathrm{R}(55)$ & 2128.8350 \\
$\mathrm{z}^{5} \mathrm{P}_{1}^{0} \rightarrow \mathrm{e}^{5} \mathrm{D}_{0}$ & 6302.5017 & $5-4 \mathrm{R}(28)$ & 2129.3799 \\
& & $7-6 \mathrm{R}(65)$ & 2140.9275 \\
& & $4-3 \mathrm{R}(28)$ & 2156.6710 \\
& & $1-0 \mathrm{R}(8)$ & 2176.2835 \\
& & $3-2 \mathrm{R}(73)$ & 2262.7267 \\
& & $3-2 \mathrm{R}(75)$ & 2264.1705 \\
$\mathrm{a}^{6} \mathrm{~S}_{5 / 2} \rightarrow \mathrm{z}^{6} \mathrm{P}_{3 / 2}^{0}$ & 4923.930 & $3-2 \mathrm{R}(78)$ & 2265.9903 \\
$\mathrm{~b}^{4} \mathrm{~F}_{9 / 2} \rightarrow \mathrm{z}^{6} \mathrm{P}_{7 / 2}^{0}$ & 4993.3527 & $1-0 \mathrm{R}(42)$ & 2273.5527 \\
$\mathrm{~b}^{4} \mathrm{~F}_{9 / 2} \rightarrow \mathrm{z}^{6} \mathrm{~F}_{7 / 2}^{0}$ & 5100.6563 & $2-1 \mathrm{R}(110)$ & 2288.8991 \\
& & $1-0 \mathrm{R}(58)$ & 2303.1663 \\
& & $1-0 \mathrm{R}(61)$ & 2307.4949 \\
& & & \\
\hline
\end{tabular}




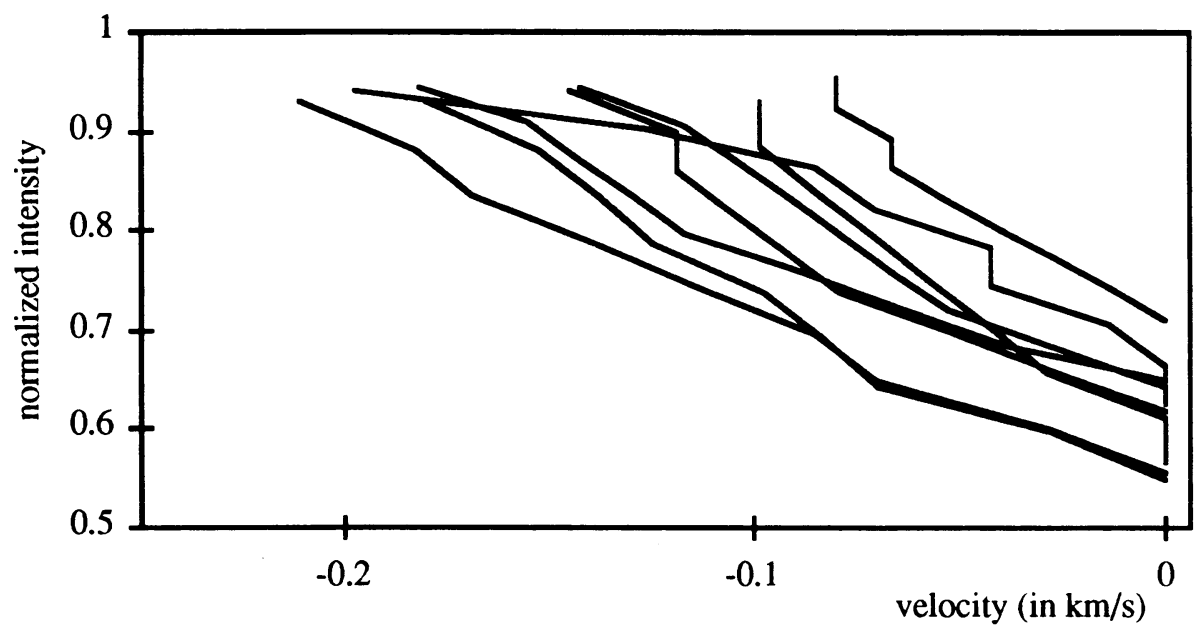

Fig. 2. Some representative examples of observed line bisectors for the $\mathrm{CO}$ lines. All bisectors have their footpoints shifted to zero.

In the infrared part of the spectrum we studied the spectra taken by the ATMOS experiment (Farmer and Norton, 1989). As not sufficient information is available to determine an absolute wavenumber scale, we cannot determine the absolute shifts of the line centers. The relative shifts show a very good correlation with the optical depth at which the line center is formed. Weaker lines (formed at larger depths) show a higher line shift than stronger lines (formed higher in the photosphere) (see also Grevesse and Sauval, 1991). For 13 CO lines that did not show any sign of blending (Table I), we determined the bisectors shown in Figure 2. They do not have the classical "C" shape as for the lines in the visible; instead the top part of the "C" seems to be missing, which is probably due to the different depths at which the continuum is formed for these wavelengths. Note that for these CO lines the observed asymmetries cannot be due to isotopic splitting - not even partially - contrary to what has been claimed for atomic lines (Kurucz, 1992).

\section{Theoretical Model}

We use a two-component model for the solar photosphere in which the hot component represents the rising granules and the cool component the descending intergranular material. We have to specify the relative surface areas of both components and their run of temperature as a function of depth. For the hot component we also specify the run of the velocity: the equation of mass conservation then gives the run of velocity for the cool component as well.

For each component the number densities of all molecules, atoms, and ions are calculated (in Local Thermodynamic Equilibrium) and the radiation transfer equation is solved for wavenumbers (or wavelengths) near the spectral lines of interest. 


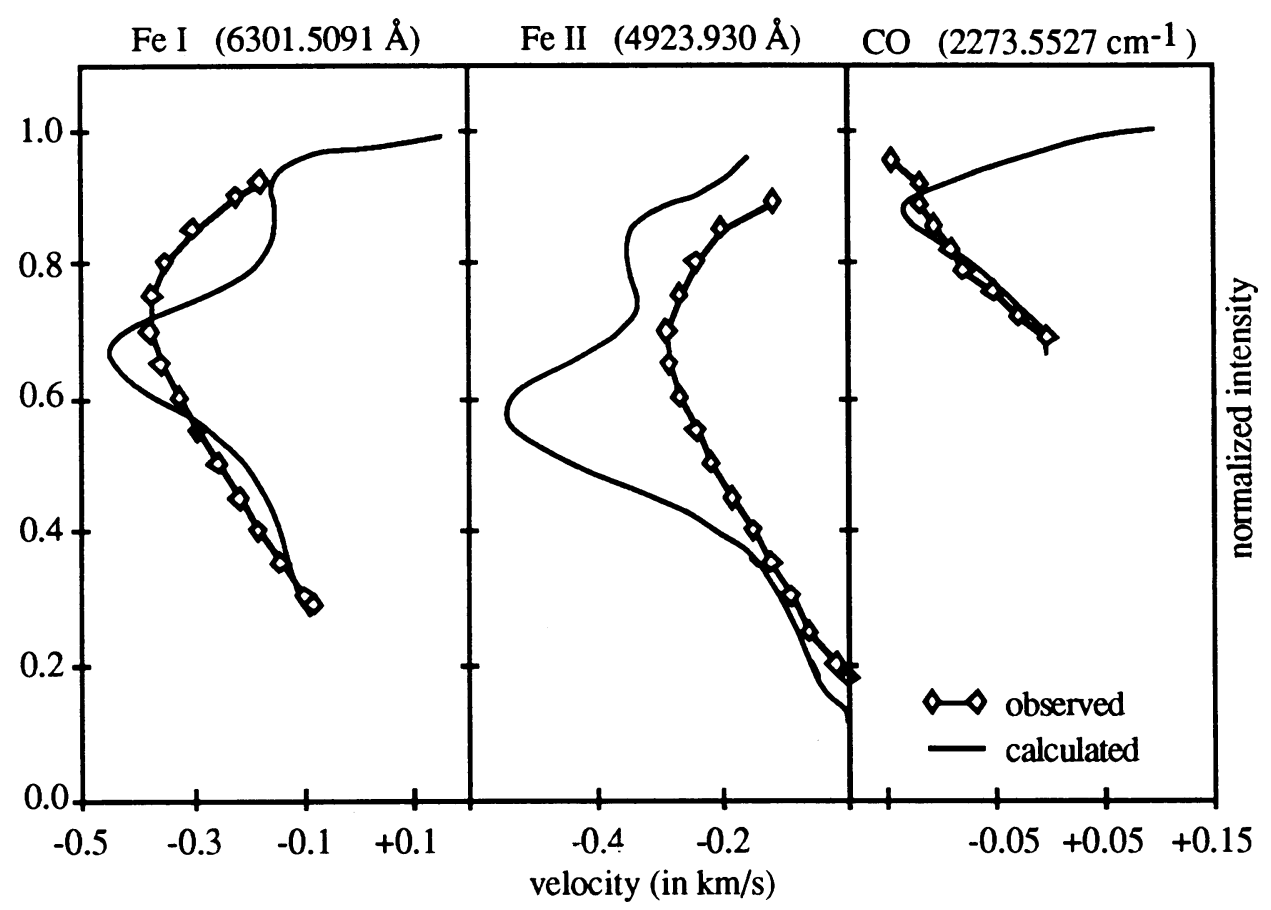

Fig. 3. The fit of our model calculation to the observed bisectors.

The sum of both line intensities (weighted by the relative surface areas) divided by the equivalent sum for the continua gives the resulting line profile. From this the bisector can be obtained and compared with the observed one.

\section{Results}

The best fit we have found so far uses the Holweger and Müller (1974) solar model photosphere for the temperature structure of the cool component. The hot component is based on this as well but the temperature was increased by a percentage that increases linearly from zero at $\log \tau_{0}=-1.3$ up to $24 \%$ at $\log \tau_{0}=+0.8$, where $\tau_{0}$ is the optical depth at $5000 \AA$ for the Holweger and Müller model. For deeper layers a constant value of $24 \%$ was taken. For the velocity law of the hot component we took an exponential function:

$$
v=1.472 \exp (-z / 150)
$$

where $v$ is the velocity in $\mathrm{km} / \mathrm{s}$ and $z$ is the geometrical depth in $\mathrm{km}$, measured from the point where $\tau_{0}=1$. The results for three typical lines are presented in Figure 3. While the agreement between theory and observations is reasonable, some improvement is needed, especially for the Fe II line. 


\section{Conclusions}

It must be stressed that the present results are only preliminary. The parameterspace is very large and has not yet been exhaustively explored. Already some conclusions can be drawn, however. The data we have for the infrared $\mathrm{CO}$ lines give complementary information not found from the lines in the visible. This might have been expected a priori because the $\mathrm{CO}$ molecule is very sensitive to temperature and $\mathrm{CO}$ lines are formed over a very large range of formation depths. A posteriori, our model calculations confirmed this, as we sometimes found models that could explain the $\mathrm{CO}$ bisectors very well, but not the Fe ones, and vice-versa. This complementary information should allow us to derive a unique solution for the run of temperature and velocity (as discussed for example, by Kaisig and Durrant, 1982).

A solar photospheric model should explain not only the line bisectors but also the observed continuum intensities, line intensities and equivalent widths. In future work we shall also look at the weaker lines - which were left out here based on a qualitative argument. Furthermore, as has been pointed out above, complementary information can be obtained by using other atoms/molecules and by studying as large a spectral range as possible.

Finally one should also note that these techniques are not limited to the sun, but $\mathrm{CO}$ lines have also been used to study turbulence in cool stars (see, e.g., Tsuji, 1991).

\section{Acknowledgements}

We thank Drs C.B. Farmer and M. Gunson (Jet Propulsion Laboratory) for permission to use the ATMOS infrared solar spectra before publication, for their hospitality and their generous help. We also thank W. Nijs for his help with implementing the computer code. All calculations were carried out on the Apollo computers at the Royal Observatory (Brussels).

\section{References}

De Jager, C.: 1959, in Handbuch der Physik LII , Springer, p. 80.

Delbouille, L., Neven, L., Roland, G.: 1973, Photometric Atlas of the Solar Spectrum from $\lambda 3000$ to $\lambda 10000$, Institut d'Astrophysique, Université de Liège.

Dravins, D., Larsson, B., Nordlund, $\AA$ : 1986, Astron. Astrophys. 158, 83.

Dravins, D., Lindegren, L., Nordlund, $\AA$ : 1981, Astron. Astrophys. 96, 345.

Farmer, C.B., Norton, R.H.: 1989, A High-Resolution Atlas of the Infrared Spectrum of the Sun and the Earth Atmosphere from Space, NASA Ref. Publ. 1224, Volume I.

Farrenq, R., Guelachvili, G., Sauval, A.J., Grevesse, N., Farmer, C.B.: 1991, J. Molec. Spectrosc. $149,375$.

Grevesse, N., Sauval, A.J.: 1991, in C. Jaschek and Y. Andrillat (eds.), The Infrared Spectral Region of Stars, Cambridge University Press, Cambridge, p. 215.

Holweger, H., Müller, E.A.: 1974, Solar Phys. 39, 19.

Kaisig, M., Durrant, C.J.: 1982, Astron. Astrophys. 116, 332.

Kurucz, R.: 1992, Astrophys. J. Letters, preprint.

Nadeau, D.: 1988, Astrophys. J. 325, 480.

Pierce, A.K., Breckinridge, J.B.: 1973, The Kitt Peak Table of Photographic Solar Spectrum Wavelengths, Contrib. No. 559, Kitt Peak National Observatory, Tucson, Arizona.

Tsuji, T.: 1991, Astron. Astrophys. 245, 203 